\title{
The first Portuguese National Health Examination Survey (2015): design, planning and implementation
}

\section{Baltazar Nunes ${ }^{1,2}$, Marta Barreto ${ }^{1,2}$, Ana P. Gil ${ }^{3}$, Irina Kislaya ${ }^{1,2}$, Sónia Namorado ${ }^{1,2}$, Liliana Antunes ${ }^{1}$, Vânia Gaio ${ }^{1,2}$, Ana J. Santos ${ }^{1}$, Ana P. Rodrigues', Joana Santos ${ }^{1}$, Rita Roquette ${ }^{1}$, Clara Alves-Alves ${ }^{4}$, Emília Castilho ${ }^{5}$, Eugénio Cordeiro ${ }^{6}$, Ana Dinis ${ }^{7}$, Tamara Prokopenko ${ }^{8}$, Ana C. Silva ${ }^{9}$, Patrícia Vargas ${ }^{10}$, Heidi Lyshol ${ }^{11}$, Carlos M. Dias ${ }^{1,2}$}

${ }^{1}$ Department of Epidemiology, Instituto Nacional de Saúde Doutor Ricardo Jorge, Av. Padre Cruz, 1649-016 Lisboa, Portugal

${ }^{2}$ Centro de Investigação em Saúde Pública, Escola Nacional de Saúde Pública, Universidade Nova de Lisboa, Lisboa, Portugal

${ }^{3}$ CICS.NOVA - Centro Interdisciplinar de Ciências Sociais, Universidade NOVA de Lisboa, Lisboa, Portugal

${ }^{4}$ Department of Public Health, Norte Regional Health Administration, Porto, Portugal

${ }^{5}$ Department of Public Health, Algarve Regional Health Administration, Faro, Portugal

${ }^{6}$ Department of Public Health, Centro Regional Health Administration, Coimbra, Portugal

${ }^{7}$ Department of Public Health, Lisbon and Tagus Valley Regional Health Administration, Lisboa, Portugal

${ }^{8}$ Department of Public Health, Alentejo Regional Health Administration, Évora, Portugal

${ }^{9}$ Autonomous Region of Madeira, Regional Government, Vice.Presidency, Funchal, Portugal

${ }^{10}$ Regional Directorate for Health, Regional Secretariat for Health, Autonomous Region of Azores, Angra do Heroísmo, Portugal

${ }^{11}$ Department of Health and Inequality, Norwegian Institute of Public Health, Oslo, Norway

Address correspondence to Baltazar Nunes, E-mail: baltazar.nunes@insa.min-saude.pt

\begin{abstract}
Background In Health Examination Surveys interview information is complemented with objective information, providing more accurate indicators than self-reported data. We report the study design, planning and implementation of the first Portuguese Health Examination Survey (INSEF).

Methods INSEF was a cross-sectional population-based study representative at regional and national level. Individuals aged between 25 and 74 years old, residing in Portugal were selected from the national health users' registry through multi-stage stratified probabilistic sampling. Sample size was set at 4200 individuals. Data was collected in primary care units and included blood pressure, height, weight, hip and waist measurements, blood collection for lipid profile, HbA1c and blood count and a general health questionnaire. European HES procedures were followed.

Results A total of 4911 individuals agreed to participate (43.9\% participation rate). Participation rate varied by region, sex and age group, being lower in Lisbon and Tagus Valley (32.8\%), for men (41.8\%) and for those aged 25-34 years old (36\%).

Conclusions INSEF has set up an experienced national and regional structure for HES implementation. Nationally representative quality epidemiological data is now available for public health monitoring, planning and research.
\end{abstract}

Keywords epidemiology, public health, methods, health examination survey

Baltazar Nunes, Assistant Researcher

Marta Barreto, Research Fellow

Ana P. Gil, Research Fellow

Irina Kislaya, Research Fellow

Sónia Namorado, Research Fellow

Liliana Antunes, Research Fellow

Vânia Gaio, Research Fellow

Ana J. Santos, Research Fellow

Ana P. Rodrigues, Medical doctor, Senior Public

Health Specialist
Joana Santos, Senior Technician

Rita Roquette, Senior Technician

Clara Alves-Alves, Medical doctor, Senior Public

Health Specialist

Emília Castilho, Senior Technician

Eugénio Cordeiro, Medical Doctor, Senior Public

Health Specialist

Ana Dinis, Medical doctor, Senior Public Health

Specialist
Tamara Prokopenko, Medical doctor, Senior Public Health Specialist

Ana C. Silva, Deputy Vice-President's Office

Patrícia Vargas, Head of Division of Planning and Quality

Heidi Lyshol, Senior Adviser

Carlos M. Dias, Director, Department of

Epidemiology 


\section{Introduction}

Population-based national health surveys are a unique source of information for in-depth knowledge on health and disease status of the population and its determinants, given that such surveys collect individual level information on various social, behavioral and health statuses. Furthermore, these national surveys allow the identification of factors that influence inequalities between and within population groups. ${ }^{1}$

In Portugal, five National Health Interview Surveys (INS) have been conducted in 1987/88, 1995/96, 1998/99, 2005/ $06^{2}$ and 2014. ${ }^{3}$ These surveys represent important tools for public health planning and interventions, essential for the development and monitoring of the National and Regional Health Plans.

Health examination surveys, in which the information collected by a detailed questionnaire is complemented with objective information measured by physical examination and laboratory tests on biological samples, provide accurate and better quality information. ${ }^{4,5}$

In Portugal, none of the population-based epidemiological studies already performed ${ }^{6-8}$ have followed the standardized methods of the European Health Examination Survey (EHES), ${ }^{9-11}$ ensuring international comparability. The only previous experience conducted in Portugal was the EHES pilot project in 2009-10 developed in Algarve, which comprised a sample of 221 individuals aged 25 years or more. ${ }^{12}$

The first Portuguese National Health Examination Survey 2015 (INSEF) was developed from the EHES pilot as part of the project, 'Improvement of epidemiological health information to support public health decision and management in Portugal. Towards reduced inequalities, improved health and bilateral cooperation.', by the National Health Institute Doutor Ricardo Jorge (INSA) in partnership with the Norwegian Institute of Public Health (NIPH), and in collaboration with the five Regional Health Administrations (ARS) and the Regional Health and Social Affairs Offices (SRAS) of the Autonomous Regions of the Azores and Madeira.

The objective of INSEF was to collect high quality epidemiological data on health status, health determinants and use of health care services, in a representative sample of residents in Portugal aged between 25 and 74 years old in 2015.

\section{Methods}

\section{Study design and population}

INSEF was planned as a cross-sectional prevalence study possible to replicate, to develop the follow-up and the re-examination of the participants in terms of morbidity and mortality.
This study was designed in order to be representative, at national and regional level, of the non-institutionalized individuals aged between 25 and 74 years old in 2015, living in Portugal for more than 12 months.

INSEF was approved by the National Commission for Data Protection, by the Ethical Committees of INSA, of the five mainland Health Administration Regions and of the two autonomous regions Health Secretaries.

All participants signed an informed consent form to perform the health examination, donate a blood sample for clinical tests and answer the health questionnaire through an interview.

Additionally the informed consent form included nonmandatory expressed permission to be re-contacted for reexamination and follow-up, to store the biological samples in the INSEF biospecimen collection, to link the survey data to the national electronic health registries and to send the participants' blood tests results to their personal general practitioner.

\section{Sample design, size, frame and procedures}

A two-stage cluster probabilistic sample stratified, by region and typology of urban area (TIPAU), was performed. In the first stage, geographical areas corresponding to national health centers catchment areas (Primary Sampling Units; PSU) were selected in each region/TIPAU stratum. In the second stage, individuals (Secondary Sampling Units; SSU) were selected by simple random sampling from the National Health Users' Registry of each selected health center.

In order to obtain similar precision for health indicators estimates, the minimum sample size was set at 600 individuals in each of the seven regions, which represent the minimum size necessary to estimate an expected prevalence of $50 \%$, with a precision of $5 \%$ for $95 \%$ confidence level, considering a design effect of 1.5. At national level, the minimum sample size was set at 4200 , oversampled to account for an expected participation rate of $40 \%$.

In each of the seven health regions (North, Center, Lisbon and Tagus Valley, Alentejo, Algarve, Azores and Madeira), seven PSU were randomly selected, with probability proportional to the PSU catchment area resident population (Fig. 1).

In the second phase, individuals were selected from the National Health Users' Registry, which covers $\sim 100 \%$ of the population residing in Portugal.

The sampling procedure was performed using the $\mathrm{R}$ package EHES sampling.

Response rates were calculated according to EHES definitions. 9 
A

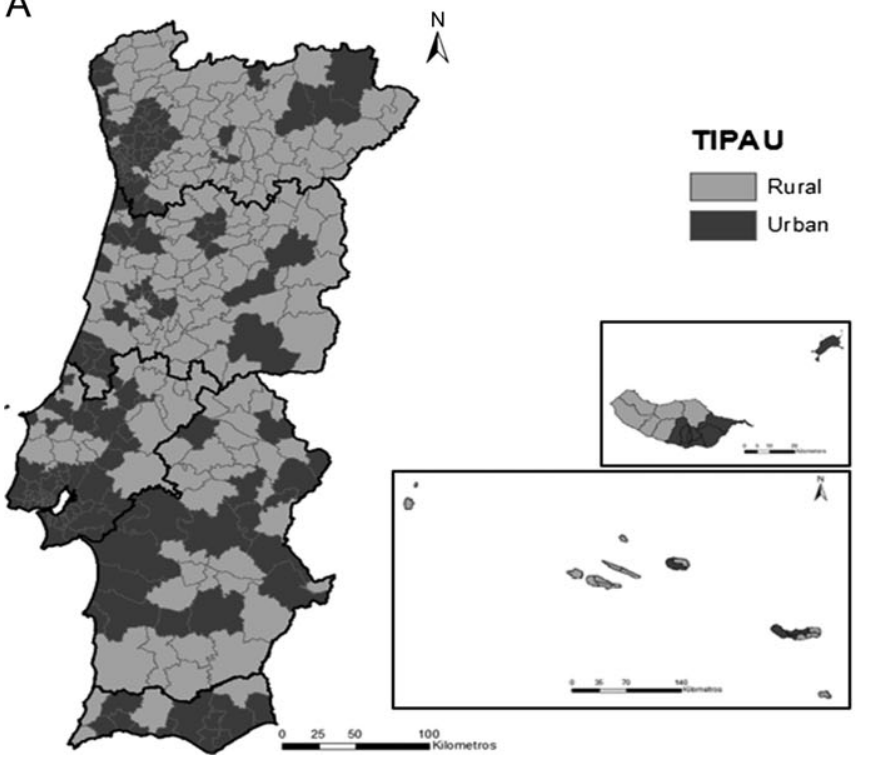

B

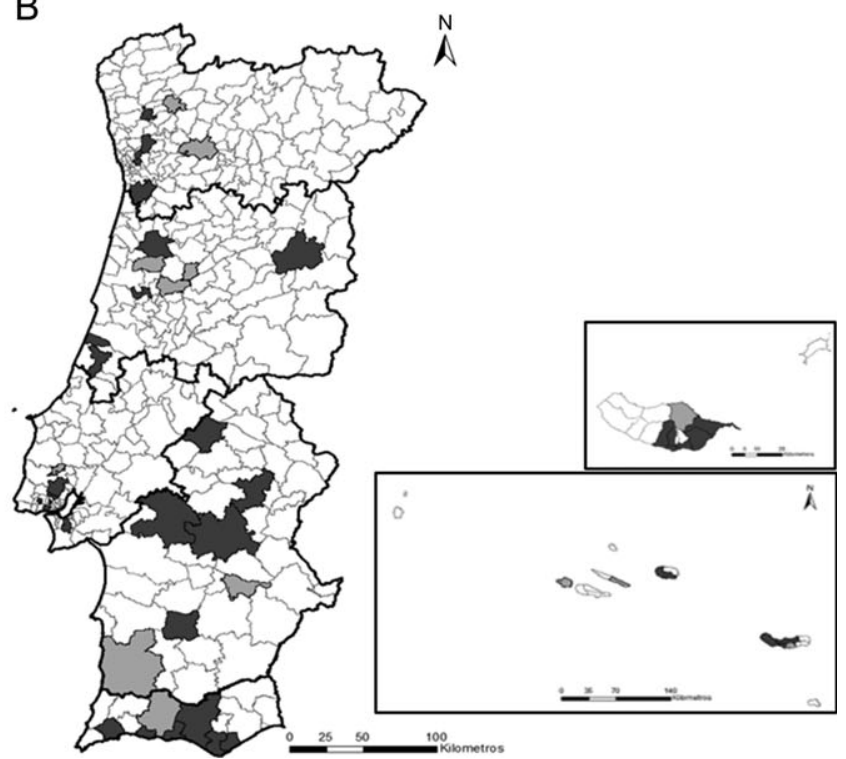

Fig. 1 Typology of urban area (TIPAU) of the total PSU $(\mathbf{A})$ and of the selected PSU (B).

\section{Instruments and procedures used for data collection}

INSEF collected data on the socio-demographic characteristics of individuals, their health and disability status, health determinants, risk factors and use of health care services, including preventive care. Questionnaire topics were selected according to the European Health Examination and National health survey recommendations. Input was also given by the Health Regions representatives and by the scientific community experts on different fields.

The survey included three components: physical examination, which comprised the measurement of blood pressure, height, weight, waist and hip circumference; blood collection for the determination of the lipid profile (total cholesterol, HDL, LDL and triglycerides), the glycated hemoglobin (HbA1c) and blood count, and an interview using a structured health questionnaire.

The health questionnaire was applied by Computer Assisted Personnel Interview using the software REDCapResearch Electronic Data Capture. ${ }^{13}$ All other information was collected using paper-based forms designed for optical reading and data entry.

Selected individuals that refused to participate were invited to answer a non-participating structured telephone questionnaire.

\section{Fieldwork and training}

Data collection was performed during 2 consecutive weeks in PSU (health centers).
The fieldwork was planned to last from February to June 2015.

Data collection was performed by 24 regional teams, each consisting of one administrative officer, two nurses (one appointed as team coordinator) and one laboratory technician or nurse to perform the blood collection and processing.

To ensure the standardization of data collection procedures, all regional team members underwent a training program that took between 21 and $28 \mathrm{~h}$.

Four operating manuals, with standard operational procedures, were provided in the training sessions. These manuals described all the examination and equipment verification procedures for the different survey stages.

\section{Invitation and data collection}

Approximately 2 weeks before starting data collection, invitation letters were mailed to the sampled individuals. About one week before the fieldwork, the team's administrative member contacted each of the selected individuals by telephone to verify eligibility criteria and schedule the appointment. Refusals were followed, when possible, by the nonparticipating questionnaire. No financial or in-kind incentives to participation were given to participants.

After arrival, participants followed a predefined circuit (Fig. 2). A nurse presented the study and asked participants to give their written informed consent. The physical examination was the first step and followed a predefined order starting with blood pressure measurement and ending with measurement of the hip and waist circumference. 


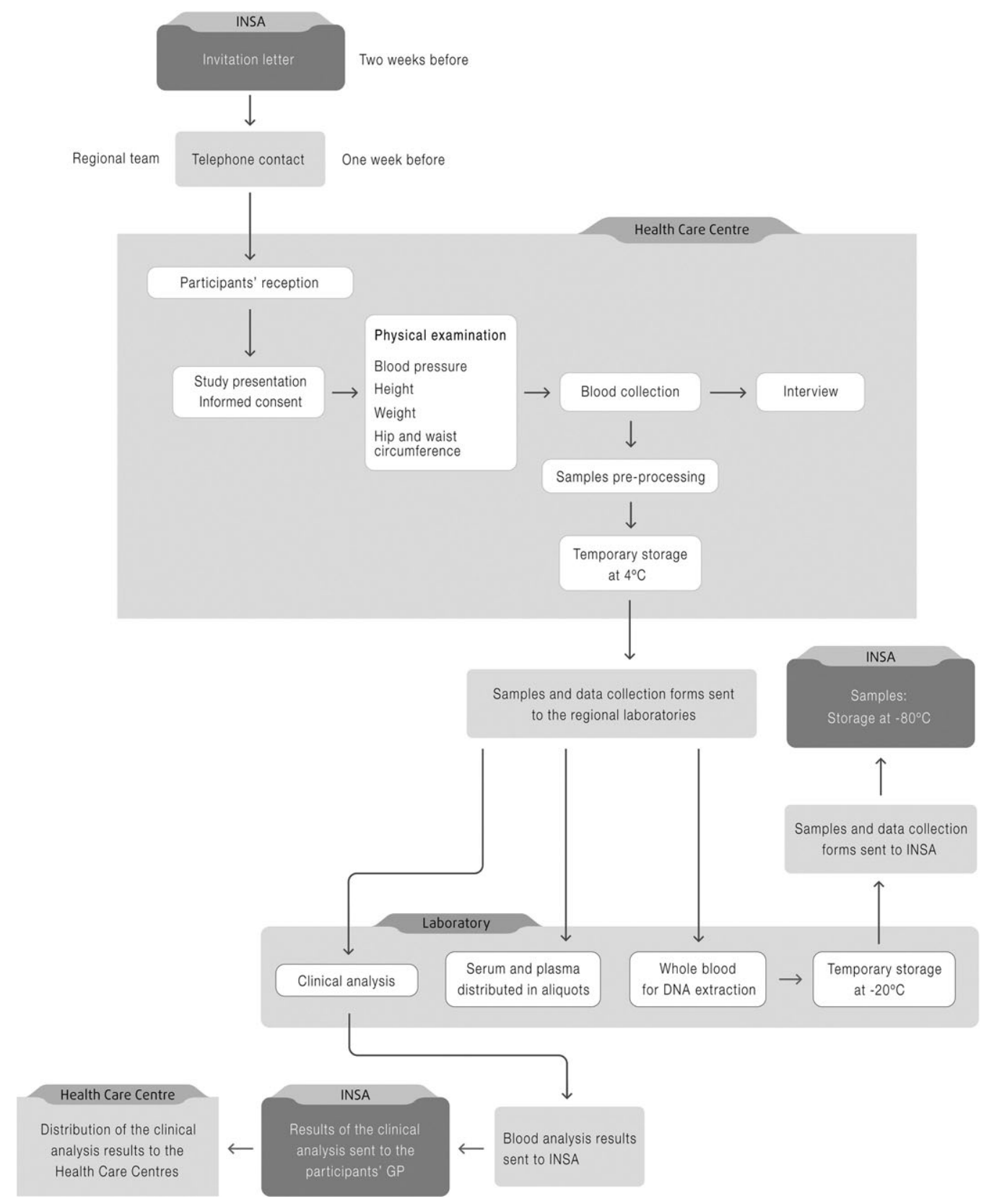

Fig. 2 Procedures flowchart.

Afterwards participants were directed for blood collection of non-fasting venous blood samples $(20 \mathrm{ml})$. Finally a structured questionnaire was applied by a nurse using CAPI in a separate room.

Blood samples were pre-processed at the health center and temporarily stored at $4^{\circ} \mathrm{C}$. At the end of each day, data collection forms and blood samples were transported to the regional laboratory in a temperature monitored refrigerated setting. At the regional laboratory, the samples were analyzed in order to determine the values of the parameters listed above. The remaining serum and plasma was distributed in aliquots and temporarily stored at $-20^{\circ} \mathrm{C}$. Whole blood samples were directly stored at $-20^{\circ} \mathrm{C}$ for later DNA extraction.

Samples were transported from regional laboratories to INSA to be stored at the INSEF biospecimen collection at $-80^{\circ} \mathrm{C}$. The results of the clinical analysis were sent to INSA and mailed to the participants' general practitioners.

All standard operational procedures for the physical exam and blood collection followed the EHES recommendations. ${ }^{10}$ 


\section{Data quality procedures}

INSEF quality procedures were based on the Plan-DoCheck-Act Cycle $^{14}$ that considers the potential for continuous improvement.

The study protocol was developed with standard operating procedures that included quality control measurements proposed for EHES. ${ }^{9-11}$ These included the protocol development, training of field teams, pilot study, equipment calibration and verification in addition to specific statistical quality control measures (end-digit distribution, equipment and interviewer's variation, duration of procedures and data checks for consistency).

External quality assessment was carried out by the NIPH and the reference center for EHES (National Institute for Health and Welfare, Finland).

The regional laboratories that participated in INSEF were included in the National External Quality Assessment Program (PNAEQ) of INSA, during fieldwork.

\section{Results}

The fieldwork was conducted between February and December 2015. It took between 2 and 5 months for each region to complete the fieldwork in the seven PSU.

\section{Response rates}

Overall, 12289 individuals were randomly selected from the 49 PSU. Of these individuals $1090(8.9 \%)$ were considered not eligible, $3415(27.8 \%)$ were not reached by the administrative and considered of unknown eligibility, a total of 7784 (63.3\%) were eligible for participation and 4911 effectively participated in INSEF.

The main non-eligibility criteria found was 'not living in the catchment area of the selected PSU or not living in Portugal for at least 12 months'.

The number of telephone contact attempts for each selected individual varied between 1 and 12; 51.0\% were reached at first contact attempt.

Of eligible individuals, 2873 were non-participants: 2104 refusals; 766 no shows and 3 did not complete $50 \%$ of the survey procedures. Of the non-participants, 1575 (54.8\%) answered the non-participant questionnaire. The main reasons for refusals concerned work related issues $(26.6 \%)$ or lack of time $(26.6 \%)$.

The overall participation rate was $43.9 \%$, varying between $32.8 \%$ in Lisbon and Tagus Valley and $56.8 \%$ in the North (Table 1). The response rate was higher in women $(45.9 \%)$ and in individuals aged between 55 and 64 years old (49.1\%). When stratified by sex, for men, the participation was higher in the oldest studied age group (65-74).

Table 1 Distribution of the total number of selected individuals according to the eligibility criteria, participation status and participation rate, by age group and region

\begin{tabular}{|c|c|c|c|c|c|c|c|c|c|c|c|c|c|}
\hline & \multicolumn{2}{|l|}{ Total } & \multicolumn{2}{|c|}{ Eligible } & \multicolumn{2}{|c|}{ Not eligible } & \multicolumn{2}{|c|}{ Unresolved } & \multicolumn{2}{|c|}{ Participant } & \multicolumn{3}{|c|}{ Participation rate } \\
\hline & $M(\mathrm{n})$ & $F(\mathrm{n})$ & $M(n)$ & $F(\mathrm{n})$ & $M(n)$ & $F(n)$ & $M(n)$ & $F(\mathrm{n})$ & $M(n)$ & $F(\mathrm{n})$ & $M(\%)$ & $F(\%)$ & Total (\%) \\
\hline Total & 6057 & 6232 & 3586 & 4198 & 622 & 468 & 1849 & 1566 & 2265 & 2646 & 41.7 & 45.9 & 43.9 \\
\hline \multicolumn{14}{|l|}{ Age group } \\
\hline $25-34$ & 1161 & 1178 & 584 & 683 & 201 & 152 & 376 & 343 & 330 & 384 & 34.4 & 37.4 & 36.0 \\
\hline $35-44$ & 1433 & 1410 & 814 & 937 & 162 & 116 & 457 & 357 & 511 & 624 & 40.2 & 48.2 & 44.2 \\
\hline $45-54$ & 1418 & 1423 & 826 & 993 & 121 & 76 & 471 & 354 & 537 & 656 & 41.4 & 48.7 & 45.1 \\
\hline $55-64$ & 1182 & 1190 & 768 & 877 & 82 & 56 & 332 & 257 & 514 & 584 & 46.7 & 51.5 & 49.1 \\
\hline $65-74$ & 863 & 1031 & 594 & 708 & 56 & 68 & 213 & 255 & 373 & 398 & 46.2 & 41.3 & 43.6 \\
\hline $35-64$ & 4033 & 4023 & 2408 & 2807 & 365 & 248 & 1260 & 968 & 1562 & 1864 & $42.5^{*}$ & $49.3^{*}$ & $45.9 *$ \\
\hline \multicolumn{14}{|l|}{ Region } \\
\hline Norte & 749 & 837 & 434 & 583 & 106 & 83 & 209 & 171 & 337 & 440 & 52.4 & 58.4 & 55.6 \\
\hline Centro & 859 & 841 & 522 & 585 & 58 & 31 & 279 & 225 & 343 & 363 & 42.8 & 44.8 & 43.8 \\
\hline LVT & 1021 & 1135 & 544 & 687 & 94 & 83 & 383 & 365 & 297 & 353 & 32.0 & 33.6 & 32.8 \\
\hline Alentejo & 771 & 806 & 521 & 588 & 47 & 35 & 203 & 183 & 318 & 372 & 43.9 & 48.2 & 46.2 \\
\hline Algarve & 947 & 875 & 540 & 544 & 56 & 55 & 351 & 276 & 313 & 331 & 35.1 & 40.4 & 37.6 \\
\hline RAM & 891 & 950 & 508 & 627 & 138 & 120 & 245 & 203 & 316 & 379 & 42.0 & 45.7 & 43.9 \\
\hline RAA & 819 & 788 & 517 & 584 & 123 & 61 & 179 & 143 & 341 & 408 & 49.0 & 56.1 & 52.6 \\
\hline
\end{tabular}

Categories eligible, not eligible, unresolved and participant were defined according to EHES definitions; $(n)$ total number of individuals. *Participation rates age-standardized according to European reference population. 
Differences in the participation rate by sex were observed in all regions except for Lisbon and Tagus Valley.

\section{Data collected}

The physical examination and interview were performed for all participants and data is available for 4911 participants. From these, blood samples were collected from 4852 $(98.8 \%)$ individuals. It was not possible to collect blood from $1.2 \%$ of the individuals due to exclusion criteria or failure to perform the blood collection in two attempts. The median time of interview and examination was 65 min (interquartile range: $56-77 \mathrm{~min}$ ).

Regarding the informed consent, 98.4\% $(n=4818)$ $98.2 \%(n=4813)$ and $97.8 \%(n=4790)$ have respectively agreed to be re-contacted for re-examination, for follow-up through data linkage and to donate extra blood to the INSEF Biospecimen Collection. Overall, 95.7\% $(n=4701)$ of the participants agreed simultaneously to all three proposals.

\section{Data quality}

The proportion of item-response missing values for the key measurements of physical examination was low, varying from $0.01 \%$ for the blood pressure measurements to $0.6 \%$ for waist circumference. For the blood analysis the lowest percentage of missing values was observed for lipid profile (total cholesterol 1.3\%) and the highest for HbA1c (2.4\%). The main reason for missing values was technical failure to collect blood. Most of blood samples (95.4\% of serum and $92 \%$ of plasma samples) were processed by regional laboratories within $24 \mathrm{~h}$ after collection.

Regarding questionnaire data, of the 283 items, four had more than $10 \%$ of missing values varying from 10.6 to $14.6 \%$. All these questions were related with data on 'time since the last consultation' or 'time since last medical exam'.

\section{Discussion}

\section{Main finding of this study}

We have established a database with biological samples and epidemiological data on health status, health determinants, use of health care and preventive health care on a population-based probabilistic sample, designed to be representative of the non-institutionalized population living in Portugal aged between 25 and 74 years old in 2015, at regional level. The implementation of INSEF in Portugal allowed setting up a national and regional structure with knowledge, experience and equipment to develop national HES.

\section{What is already known on this topic}

In Portugal, before the National Health Examination Survey implemented in 2015, all data on health status of the population were collected using health interview surveys. Until then, Health Examination Surveys were only developed for specific conditions such as hypertension, obesity and diabetes. $^{6-8}$ None of these disease specific surveys included a wide spectrum of health related information. In addition, they were highly heterogeneous and provided noncomparable data, since they used very different methods and questionnaires. Consequently, there was no objective information available in a Portuguese population sample including the health status, health determinants and use of health care. The available information was fragmented, not harmonized and therefore not comparable with other countries' data and which is representative from a south European population.

\section{What this study adds}

INSEF constitutes an important resource for planning, monitoring and evaluating national and regional health programs. It will also be used for population-based research, given that encompasses epidemiological data linked to a biospecimen collection and the possibility for follow-up by re-examination or data linkage.

In this study, in addition to the informed consent given to perform the core measurements, participants were also asked to give informed consent to donate a blood sample to the INSEF biospecimen collection, to be contacted for future re-examination and interviews and to link the information collected with the official electronic health records. The great majority of the participants have consented to all three proposals, increasing the potential to develop longitudinal studies to answer epidemiological research questions in the future.

INSEF data can also be for international epidemiological research, given that it was collected using the standardized procedures of EHES. ${ }^{9-11}$

\section{Limitations of this study}

The fact that the participation rate depended on the age, sex and region of residence could represent a potential selection bias, which was reduced by statistical adjustment of the sampling weights according to the non-participation rate. Also, imperfections of the sampling frame were reduced by calibrating the non-participation adjusted weights to estimates of the Portuguese population in $2014 .^{15}$

Initially, the fieldwork was planned to take place during the first semester of 2015 in all the seven regions 
simultaneously. Nevertheless, due to logistic and administrative constraints the fieldwork in three regions (Madeira Autonomous Region, Azores Autonomous Region and Lisbon and Tagus Valley Region) occurred between June and December 2015. This fact may have produced a differential information bias given that parameters such as weight, cholesterol, blood pressure may present seasonal differences. $^{16-18}$ However, this potential bias can be evaluated in specific data analysis for sensitivity.

\section{Conflict of interest}

The author's state that there are no conflicts of interest to be declared.

\section{Acknowledgements}

The authors are grateful to all the professionals involved in the INSEF fieldwork and to all the INSEF participants.

\section{Funding}

The Portuguese National Health Examination Survey 2015 (INSEF) was developed as part of the Pre-defined project of the Public Health Initiatives Program (reference 000039_ PDP), "Improvement of epidemiological health information to support public health decision and management in Portugal. Towards reduced inequalities, improved health, and bilateral cooperation", that benefits from a $1.500 .000 €$ Grant from Iceland, Liechtenstein and Norway through the European Economic Area Financial Mechanism (EEA Grants), 85\%, and from the Portuguese Government, 15\%.

\section{References}

1 Malta DC, Leal M, do C et al. Inquéritos Nacionais de Saúde: experiência acumulada e proposta para o inquérito de saúde brasileiro. Rev Bras Epidemiol 2008;11:159-67. doi:10.1590/S1415$790 \times 2008000500017$.

2 Dias CM. 25 anos de Inquérito Nacional de Saúde em Portugal. Rev Port Saúde Pública 2009;27:51-60.

3 Instituto Nacional de Estatística. (2016) Inquerito Nacional de Saúde 2014. INE, Lisboa.
4 Oyebode $\mathrm{O}$, Mindell JS. A review of the use of health examination data from the Health Survey for England in government policy development and implementation. Arch Public Health 2014;72:24. doi:10.1186/2049-3258-72-24.

5 Tolonen H, Giampaoli S, Kuulasmaa K et al. Blood pressure profiles, and awareness and treatment of hypertension in Europeresults from the EHES Pilot Project. Public Health 2016. doi:10. 1016/j.puhe.2015.10.035.

6 Polonia J, Martins L, Pinto F et al. Prevalence, awareness, treatment and control of hypertension and salt intake in Portugal. $J$ Hypertens 2014;32:1211-21. doi:10.1097/HJH.0000000000000162.

7 Sardinha LB, Santos DA, Silva AM et al. Prevalence of overweight, obesity, and abdominal obesity in a representative sample of Portuguese adults. PLoS One 2012;7:e47883. doi:10.1371/journal. pone. 0047883 .

8 Gardete-Correia L, Boavida JM, Raposo JF et al. First diabetes prevalence study in Portugal: PREVADIAB study. Diabet Med 2010; 27:879-81. doi:10.1111/j.1464-5491.2010.03017.x.

9 Tolonen H. (2013a) EHES Manual. Part A. Planning and preparation of the survey.

10 Tolonen H. (2013b) EHES Manual. Part B. Fieldwork procedures.

11 Tolonen H. (2013c) EHES Manual. Part C. European level collaboration.

12 Kuulasmaa K, Tolonen H, Koponen $\mathrm{P}$ et al. An overview of the European Health Examination Survey Pilot Joint Action. Arch Public Health 2012;70:20. doi:10.1186/0778-7367-70-20.

13 Harris PA, Taylor R, Thielke R et al. Research electronic data capture (REDCap) - a metadata-driven methodology and workflow process for providing translational research informatics support. J Biomed Inform 2009;42:377-81. doi:10.1016/j.jbi.2008.08.010.

14 McComb M A. The quality toolbox. Technometrics 2008;50:408-8.

15 INE. (2014) População residente $\left(\mathbf{N}^{\circ}{ }^{\circ}\right)$ por Local de residência (NUTS-2013), Sexo e Grupo etário; Anual, 2014. www.ine.pt

16 Arnesdatter Hopstock L, Gerard Barnett A, Harald Bønaa K et al. Seasonal variation in cardiovascular disease risk factors in a subarctic population: the Tromsø Study 1979-2008. J Epidemiol Community Health 2013;67:113-8. doi:10.1136/jech-2012-201547.

17 Marti-Soler H, Gubelmann C, Aeschbacher S et al. Seasonality of cardiovascular risk factors: an analysis including over 230000 participants in 15 countries. Heart 2014;100:1517-23. doi:10.1136/ heartjnl-2014-305623.

18 Liu B, Taioli E. Seasonal variations of complete blood count and inflammatory biomarkers in the US population-analysis of NHANES data. PLoS One 2015;10:1-10. doi:10.1371/journal.pone. 0142382 